\title{
Ultra-wide bandwidth backscatter modulation: processing schemes and performance
}

\author{
Davide Dardari ${ }^{1 *}$, Francesco Guidi ${ }^{1,2}$, Christophe Roblin $^{2}$ and Alain Sibille ${ }^{2}$
}

\begin{abstract}
Future advanced radio-frequency identification (RFID) systems are expected to provide both identification and high-definition localization of objects with improved reliability and security while maintaining low power consumption and cost. Ultrawide bandwidth (UWB) technology is a promising solution for next generation RFID systems to overcome most of the limitations of current narrow bandwidth RFID technology, such as reduced area coverage, insufficient ranging resolution for accurate localization, sensitivity to interference, and scarce multiple access capability. In this article, the UWB technology is applied to passive RFID relying on backscatter modulation. A signaling structure with clutter and interference suppression capability is proposed and analyzed. The potential performance is investigated in terms of range/data rate trade-off, clutter suppression, and multiple access capability using experimental data obtained in both the controlled and realistic environments.
\end{abstract}

Keywords: UWB, RFID, backscatter modulation, clutter suppression

\section{Introduction}

Radio-frequency identification (RFID) technology for use in real-time object identification is facing rapid adoption in several fields, such as logistic, automotive, surveillance, automation systems, etc. [1]. A RFID system consists of readers and tags applied to objects. The reader interrogates the tags via a wireless link to obtain the data stored on the tags. The cheapest RFID tags with the largest commercial potential are passive or semi-passive, where the energy necessary for tag-reader communication is harvested from the reader's signal or the surrounding environment [2].

Future advanced RFID systems are expected to provide both reliable identification and high-definition localization of tags at submeter level. New important requirements, such as accurate real-time localization, high security, large numbers of tag management, in addition to extremely low power consumption, small size, and low cost, will be mandatory [3]. Unfortunately, most of these requirements cannot be fulfilled completely by the current first and second generation RFID or wireless sensor network (WSN) technologies such as

\footnotetext{
* Correspondence: ddardari@ieee.org

${ }^{1}$ WiLAB, Dipartimento di Elettronica, Informatica e Sistemistica (DEIS),

University of Bologna at Cesena, Via Venezia 52, Cesena (FC) 47521, Italy Full list of author information is available at the end of the article
}

those based on ZigBee standard [4-6]. In fact, RFID systems using standard continuous wave $(\mathrm{CW})$-oriented communication in the ultra-high frequency (UHF) band have an insufficient range resolution to achieve accurate localization, are affected by multipath signal cancellation (due to the extreme narrow bandwidth signal), are very sensitive to narrowband interference and multi-user interference, and have an intrinsic low security [1,7-9]. Although some of these limitations, such as security and signal cancellation due to multipath, are going to be reduced or overcome in future versions of UHF RFID systems $[10,11]$, a technology change is required to fully satisfy new applications requirements, especially those related to high-definition localization at the submeter level.

A promising wireless technique for next generation RFID is the ultrawide bandwidth (UWB) technology characterized, in its impulse radio UWB (IR-UWB) implementation, by the transmission of subnanosecond duration pulses [12]. According to the Federal Communications Commission (FCC) definition, UWB emissions have a very large fractional bandwidth (greater than 0.2) or a bandwidth larger than $500 \mathrm{MHz}$ enabling us to resolve multipath, penetrating many materials with extraordinary localization precision based on time-ofarrival (TOA) estimation of the signal. The potential

\section{SpringerOpen ${ }^{\odot}$}

(c) 2011 Dardari et al; licensee Springer. This is an Open Access article distributed under the terms of the Creative Commons Attribution License (http://creativecommons.org/licenses/by/2.0), which permits unrestricted use, distribution, and reproduction in any medium, provided the original work is properly cited. 
advantages of UWB include, but are not limited to, low power consumption at the transmitter side, extremely accurate ranging and positioning capability at the submeter level, robustness to multipath (better area coverage), low detection probability (higher security), and large number of devices operating and co-existing in small areas (efficient multiple channel access and interference mitigation) [8,13-16].

Thanks to their low power consumption, IR-UWB transmitters can be adopted successfully for both active and passive tags. UWB has been proposed to realize low consumption and low complexity active radio-frequency (RF) tags for precision asset location systems [17]. Recently, some commercial proprietary real time locating systems (RTLSs) have been introduced based on tags emitting UWB pulses with extremely low duty cycles to ensure high battery duration. Another example of low complexity tag architecture is given in [18] where the concept of a UWB-based pseudo-random active reflector, which does not require the presence in the tag of a modulator and demodulator, is introduced.

When tag cost, size, and power consumption requirements become particularly stringent, passive or semi-passive tag solutions have to be taken into consideration. Communication with passive tags usually relies on backscatter modulation where the antenna reflection properties are changed according to information data, even though the tag's control logic and memory circuits have still to be energized to have the tag working properly [2]. Typically, passive RFID tags obtain the necessary power to operate from the RF signal sent by the reader. As a consequence, in conventional UHF RFID systems the corresponding operating range is usually no more than 7-8 $\mathrm{m}$ with a transmission power level of 2-4 W [19]. Unfortunately, owing to regulatory constraints, the transmission power allowed for UWB devices is below $0 \mathrm{dBm}$ [20]. This means that sufficient power cannot be derived from the received UWB signal to power up a remote tag at a significant distance. Besides the adoption of semipassive tags, where the control logic is battery powered, a promising possibility for retrieving the necessary energy is to adopt energy scavenging techniques which, in many cases, provide sufficient power (about $1 \mu \mathrm{W}$ ) for the control logic [21].

Recently, some applications of the UWB technology in tags based on backscatter modulation have been proposed. In [22], a hybrid UWB architecture is illustrated where the reader broadcasts narrowband RF signals which carry commands, the clock, and energy to the tags, whereas conventional UWB transmission is applied to the reverse link. Unfortunately, those authors do not provide results in terms of communication performance. The idea of passive tags based on UWB backscatter signaling is proposed in [23] in case of an ideal scenario where neither clutter nor interference is present. Preliminary studies can be found in [24-26] where a flexible tag architecture as well as a backscatter signaling scheme robust to the presence of clutter (i.e., reflections coming from surrounding objects) are presented. However, UWB RFID solutions based on backscatter modulation have not been investigated in realistic scenarios, and the study is in its embryonic stage. It is worth mentioning that a new European Project, namely SELECT, has recently started to investigate the feasibility of the passive UWB technology to be used as add-on in conventional UHF RFID tags for high-accuracy positioning purposes [27].

This article introduces the basic principles of backscatter communication using UWB signals for application to RFID and RTLSs. The main challenges, such as the presence of strong clutter, multi-user interference (MUI), and the absence of common synchronization between tags and readers, are discussed. Then, a tagand-reader architecture and related signal-processing schemes are proposed with the purpose to suppress the effect of clutter and allow multiple access (multi-tag). An experimental measurement campaign performed both in controlled (anechoic chamber) and realistic (laboratory) environments, to characterize the round trip channel and antenna behavior, is described. Starting from experimental data, the performance of the proposed architecture is assessed in terms of range/data rate trade-off, clutter suppression, and multiple access capability.

This article is organized as follows: In Section 2, the principle of backscatter propagation of UWB antennas and the measurement campaign set-up are illustrated. The proposed architecture for tag and reader is described and analyzed in Section 3, whereas in Section 4 , the performance is evaluated using experimental data. Some conclusions are given in Section 5.

\section{UWB Backscatter Propagation}

To design dedicated architectures for backscatter communication, it is fundamental to understand the basic electromagnetic (e.m.) mechanisms underlying the interaction between the reader's and tag's antennas. As previously mentioned, backscatter modulation consists of changing the antenna reflection properties according to information data [2]. In fact, when an e.m. wave encounters an antenna, it is partially reflected back depending on antenna configuration. The antenna scattering mechanism is composed of structural and antenna mode scatterings $[28,29]$. The structural mode occurs owing to the antenna's given shape and material and is independent from how the antenna is loaded. On the other hand, antenna mode scattering is a function of the antenna load, and thus, data can be sent back to the reader 
through a proper variation of the antenna load characteristic without requiring a dedicated power source (backscatter modulation). ${ }^{\mathrm{a}}$ This property is currently adopted in traditional passive UHF RFID tags based on CW signals to carry information from the tag to the reader.

As compared to the extensive investigations of UHF RFIDs (see [2,29-31]), further investigation of backscatter properties when operating with UWB signals, especially in realistic environments, is needed [26,32,33]. For example, in [34], the effect of metallic objects located nearby the tag is addressed both via simulations and measurements.

\section{A. UWB antenna backscattering}

When a UWB pulse is transmitted and UWB antennas are employed, the reflected signal takes the form shown in Figure 1. The structural and antenna mode scattering components are plotted separately for convenience. The antenna mode-scattered signal can be varied according to the antenna load, $Z_{L}$, whereas the scattering of the structural mode remains the same. Among the various possibilities, three particular choices are of interest for passive UWB RFID: $Z_{L}=0$ (short circuit), $Z_{L}=\infty$ (open circuit), and $Z_{L}=Z_{\mathrm{A}}^{*}$ (matched load), where $Z_{\mathrm{A}}$ is the antenna impedance. Ideally, antenna mode-scattered waveforms have a phase difference of $180^{\circ}$ between the case of open- and short-circuit loads, whereas no antenna mode scattering exists in the case of a perfectly matched load. In UWB antennas, the structural mode component takes a significant role in the total scattered signal; in fact, it is typically 1 or 2 orders of magnitude higher than that of the antenna mode [25,32]. In addition, signals scattered by the surrounding environment (clutter) are inevitably present and superimposed on the useful signal. In general, it is expected that the clutter and the antenna structural mode scattering have a significant impact at the reader's antenna, thus making the detection of the antenna mode-scattered signal (which carries data) a main issue in passive UWB RFID systems. This has not yet been widely addressed, and for this purpose, ad hoc robust backscatter modulation schemes will be designed (as illustrated in Section 3).

\section{B. The round-trip channel transfer function}

To analyze the performance of backscatter modulation schemes, a proper model for the reader-tag-reader interaction is needed.

Consider a reference scenario as shown in Figure 1 where a couple of UWB antennas, acting as tag and reader, located at distance $d$ are present. In the simple case of linear polarization of each antenna, the far-field

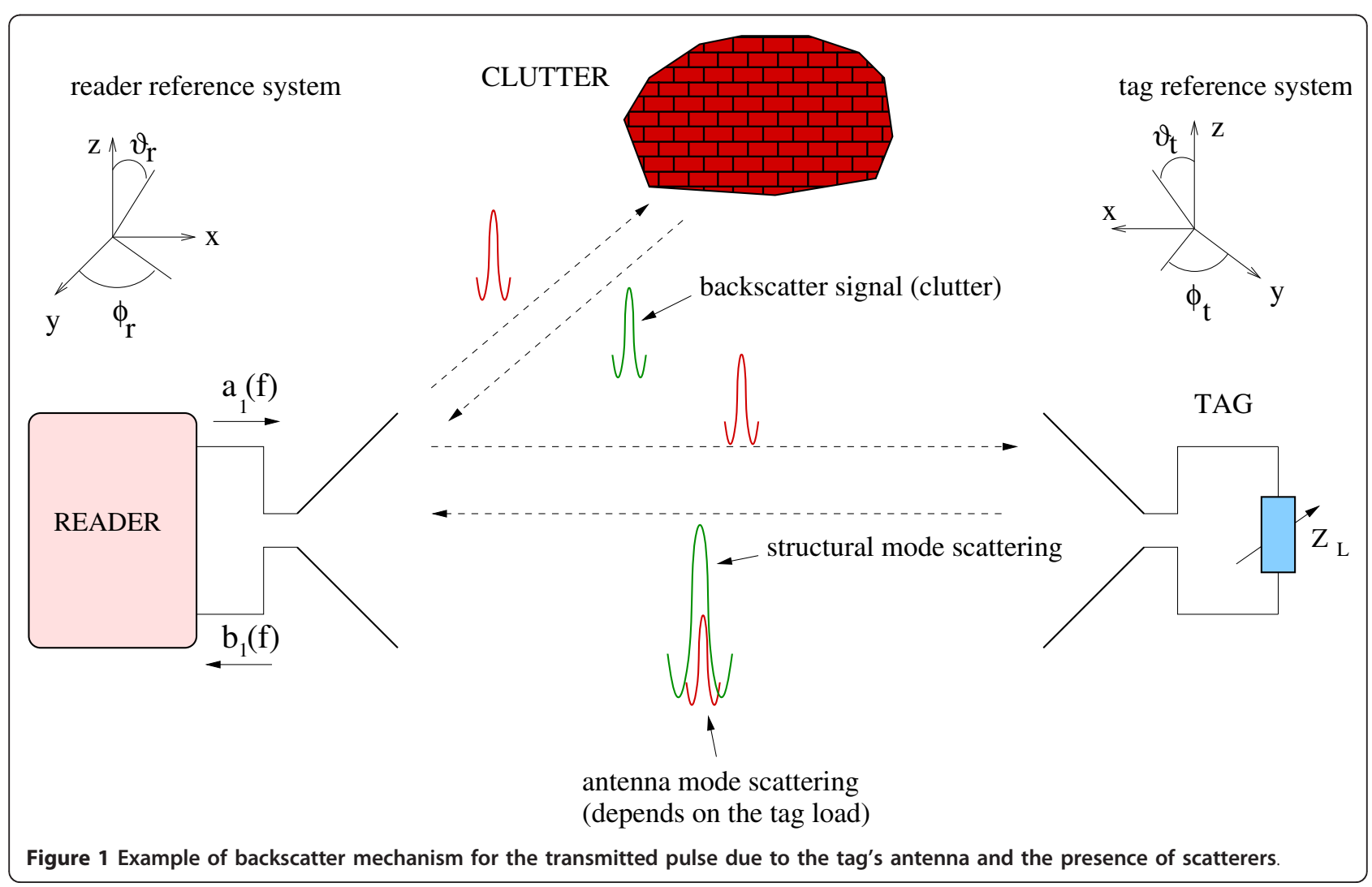


radiated from the reader and incident to the tag can be expressed as

$$
E_{\text {inc }}\left(f ; d, \boldsymbol{\Theta}_{\mathrm{r}}\right)=\frac{e^{-J^{k d}}}{d} \cdot \sqrt{\frac{\eta_{0}}{4 \pi}} \cdot H_{\text {reader }}^{\mathrm{T}}\left(f ; \boldsymbol{\Theta}_{\mathrm{r}}\right) \cdot a_{1}(f),
$$

where $\lambda$ is the wavelength, $k=2 \pi / \lambda, \eta_{0}$ is the free space impedance, $a_{1}(f)$ is the incident wave at the reader's port, $\boldsymbol{\Theta}_{\mathrm{r}}=\left(\theta_{\text {reader }}, \varphi_{\text {reader }}\right)$ is the reader orientation, and $H_{\text {reader }}^{\mathrm{T}}\left(f ; \boldsymbol{\Theta}_{\mathrm{r}}\right)$ is the antenna's transfer function in the transmitting mode of the reader [35].

The e.m. wave at the tag's antenna is partially backscattered according to the antenna's scattering characteristics which depend on the antenna load (different load configurations will be referred to as tag status $X$ ) and reader-tag orientation in the 3D space $\boldsymbol{\Theta}=\left\{\boldsymbol{\Theta}_{\mathrm{r}}, \boldsymbol{\Theta}_{\mathrm{t}}\right\}$, with $\boldsymbol{\Theta}_{\mathbf{t}}=\left(\theta_{\text {tag }}, \varphi_{\text {tag }}\right)$ being the tag orientation. The antenna mode component of the received backscatter signal at reader's antenna port is given by

$$
b_{1}(f ; d, X, \boldsymbol{\Theta})=H_{\text {reader }}^{\mathrm{R}}\left(f ; \boldsymbol{\Theta}_{\mathrm{r}}\right) \cdot \sqrt{\frac{4 \pi}{\eta_{0}}} \cdot E_{\mathrm{SC}}^{(\mathrm{a})}(f ; d, X, \boldsymbol{\Theta}),
$$

where $H_{\text {reader }}^{\mathrm{R}}\left(f ; \boldsymbol{\Theta}_{\mathrm{r}}\right)$ is the reader's antenna transfer function in receiving mode, and $E_{\mathrm{SC}}^{(\mathrm{a})}(f ; d, X, \Theta)$ is the component of the field scattered by the tag due only to the antenna mode. Therefore, we can express the tag's transfer function as

$$
H_{\mathrm{tag}}(f ; \boldsymbol{\Theta}, X)=\frac{4 \pi d}{\lambda} \cdot \frac{E_{\mathrm{SC}}^{(\mathrm{a})}(f ; d, X, \boldsymbol{\Theta}) \cdot e^{j k d}}{E_{\mathrm{inc}}\left(f ; d, \boldsymbol{\Theta}_{\mathrm{r}}\right)} .
$$

In particular, it can be shown that the amplitude characteristic of $H_{\text {tag }}(f ; \boldsymbol{\Theta}, X)$ can be expressed as

$$
\left|H_{\mathrm{tag}}(f ; \boldsymbol{\Theta}, X)\right|=\sqrt{\frac{4 \pi \sigma(f ; \boldsymbol{\Theta}, X)}{\lambda^{2}}},
$$

where $\sigma(f ; \boldsymbol{\Theta}, X)$ is the radar cross section of the tag. It turns out that this transfer function depends on the reader and local tag orientation $\boldsymbol{\Theta}$, but it is not dependent on the distance, since it relates the incoming plane wave complex amplitude at the tag to the far field radiated spherical wave. It is, for example, easy to prove that $H_{\text {tag }}(f ; \boldsymbol{\Theta}, X)=1$ for a lossless tag re-radiating isotropically. By considering the relationship between the transmitting and receiving modes of the reader

$$
H_{\text {reader }}^{\mathrm{R}}\left(f ; \boldsymbol{\Theta}_{\mathrm{r}}\right)=-\jmath \frac{\lambda}{4 \pi} H_{\text {reader }}^{\mathrm{T}}\left(f ; \boldsymbol{\Theta}_{\mathrm{r}}\right)
$$

we finally obtain the round-trip transfer function for linear polarized antennas:

$$
\begin{aligned}
H(f ; d, X, \boldsymbol{\Theta})= & \frac{b_{1}(f ; d, X, \boldsymbol{\Theta})}{a_{1}(f)}=\left[H_{\mathrm{reader}}^{\mathrm{T}}\left(f ; \boldsymbol{\Theta}_{\mathrm{r}}\right)\right]^{2} \\
& \cdot\left(-J\left(\frac{c}{f \cdot 4 \pi \cdot d}\right)^{2} e^{-J \frac{2 \pi f}{c} 2 d}\right) \cdot H_{\mathrm{tag}}\left(f ; \boldsymbol{\Theta}_{\mathrm{t}}, X\right) .
\end{aligned}
$$

Note from (6) that, in free-space propagation conditions, the channel gain decreases with the distance $d$ of an exponent factor of 2 instead of 1 as happens in conventional communication links.

In a UWB RFID system the reader's antenna emits typically a very short pulse $g(t)$. We denote $w(t ; d, X, \boldsymbol{\Theta})$ the backscattered signal, received back by the reader's antenna, because of the tag's antenna mode, shape and energy of which are a function of the tag status $X$ (open, short, loaded) as well as of $\boldsymbol{\Theta}$. In the frequency domain, it is

$$
W(f ; d, X, \boldsymbol{\Theta})=G(f) H(f ; d, X, \boldsymbol{\Theta}) \text {. }
$$

In a more realistic scenario, where several scatterers might be present, $H(f ; d, X, \boldsymbol{\Theta})$ must also account for multipath components arising due to reflections. As of now, neither statistical nor deterministic models for UWB backscatter round-trip channel with multipath are present in the literature. For this reason, in the numerical results, measured data collected in a realistic indoor scenario, described in the next section, will be used to assess the performance of the proposed UWB RFID system.

\section{Measurement campaign set-up}

Experimental data were collected in two different scenarios in ENSTA-ParisTech laboratory. The first scenario is in a controlled environment consisting of an anechoic chamber. Data collected can be successfully utilized to characterize the UWB antenna backscatter properties and to assess the system performance in ideal conditions. The second scenario (shown in Figure 2) is a realistic indoor environment consisting of a laboratory with furniture and having dimensions $5.13 \times 4.49 \mathrm{~m}^{2}$. In this case, the data collected enable the performance characterization in close-to-reality conditions.

In both scenarios, the backscattering signals from an UWB antenna were measured in the frequency domain by means of a vector network analyzer in the $2-12 \mathrm{GHz}$ band with steps of $5 \mathrm{MHz}$. Two Horn Lindgren 3117 antennas were employed as reference antennas. They were placed in a quasi-monostatic configuration, separated by $18 \mathrm{~cm}$, guaranteeing a high isolation between the transmit and receive channels as shown in Figure 2. Two kinds of UWB scattering antennas were tested, namely, a balanced antipodal Vivaldi (BAV) and a monopole dual feed stripline (DFMS) antennas [36]. In particular, the DFMS is a small planar antenna of dimensions $40 \times 24 \times 3 \mathrm{~mm}^{3}$ characterized by moderately directional and non-dispersive properties, which make it quite suitable for RFID tags [37]. Three different load conditions (open-circuit, short-circuit, and matched) and several antenna orientations were considered. Inside the anechoic chamber, the antenna under test was placed on a 3D positioner at distance $d_{\text {ref }}=1.46 \mathrm{~m}$ (DFMS) and $d_{\text {ref }}=1.44 \mathrm{~m}$ (BAV) from the reference 


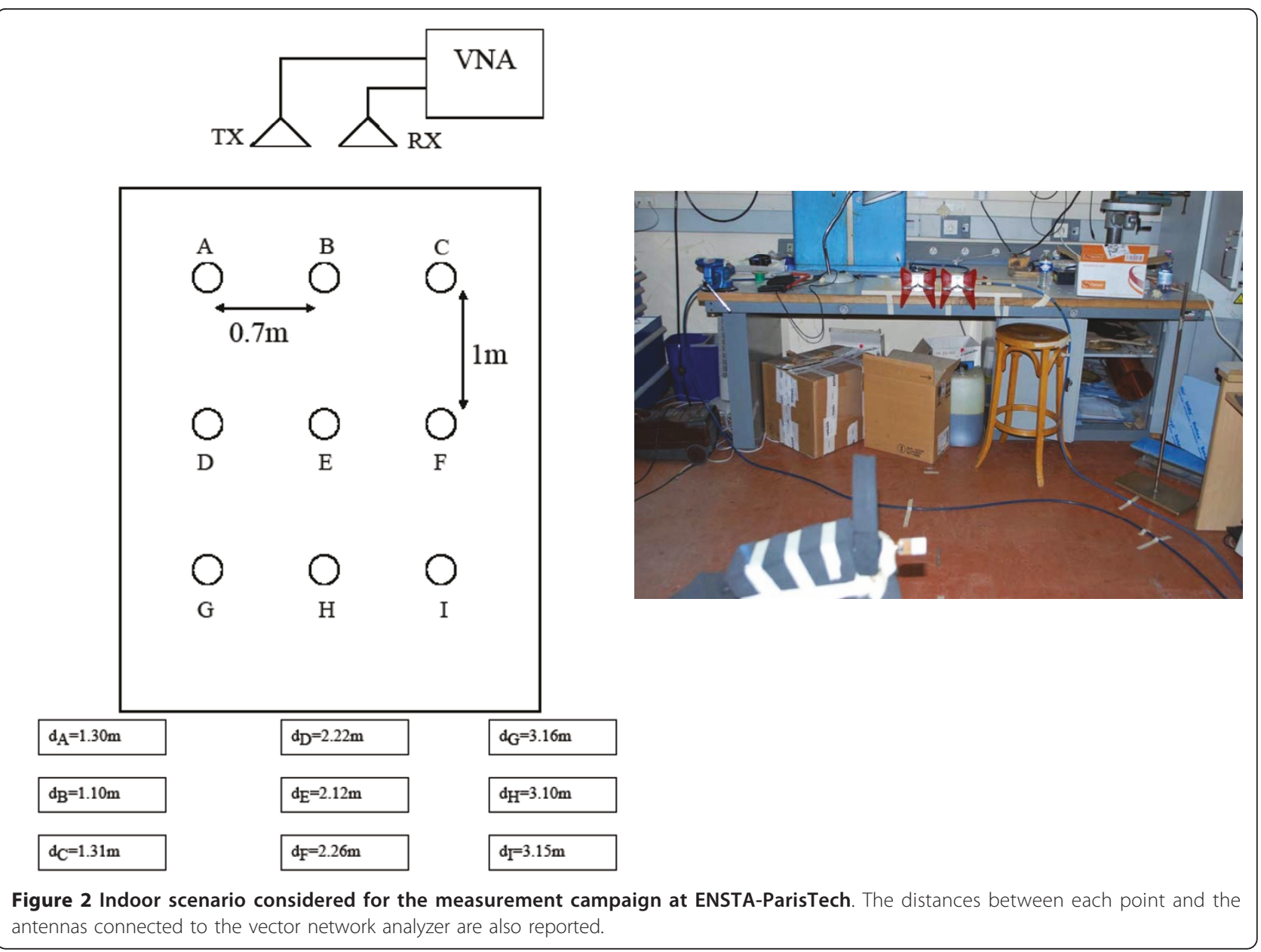

antennas dressed with absorbers to create a quasifreespace condition. The laboratory environment consisted of a room of dimension $5.13 \times 4.49 \mathrm{~m}^{2}$, where a rectangular grid of nine points as shown in Figure 2, spaced out of about $1 \mathrm{~m}$ in depth and $70 \mathrm{~cm}$ in width, was defined. The tag DFMS antenna was positioned alternatively in each point on a vertical support still dressed with absorbers. For both cases, a simple data post-processing was performed to obtain the antenna backscattering response from the measured parameter $S_{21}=H\left(f ; d_{\text {ref }}, X, \boldsymbol{\Theta}\right)$. The collected data were first windowed in the frequency domain to avoid ringing effect. Then, applying the inverse Fourier transform, the signal in the time domain was derived. In the post-processing phase, the transmitted pulse has been chosen to obtain a transmitted signal compliant with the 3.1-10.6 GHz FCC mask. Specifically, the 6th derivative Gaussian monocycle has been considered [15].

Figure 3a shows the backscattered signal in the anechoic chamber for open- and short-load conditions. In order to easily discriminate in the time domain the structural mode from the antenna mode, a $50 \Omega$ coaxial cable (delay line) of electric length $40 \mathrm{~cm}$ was inserted between the tag antenna and the load. The structural and antenna modes can be clearly distinguished, where only the latter depends on the antenna load. In particular, the difference of $180^{\circ}$ for the antenna mode scattering between the two load conditions is clearly evidenced. It has to be remarked that the delay line used for measurements is not required in a real system implementation as the communication scheme proposed in this article is able to discriminate between the antenna and structural mode components, even if they are time overlapped, as will be illustrated in the next section.

In Figure $3 \mathrm{~b}$, an example of backscattered signal received in the laboratory scenario from the tag placed at location $H$ (distance $3.10 \mathrm{~m}$ ) is reported. As can be noted, several clutter components (including the antenna structural mode) are present. Figure 3c shows the antenna mode backscattered signal (of interest) after clutter removal. Owing to its small amplitude, it turns out to be completely buried within the clutter component. The presence of some echoes received after the first direct path is also clearly seen and can be ascribed 

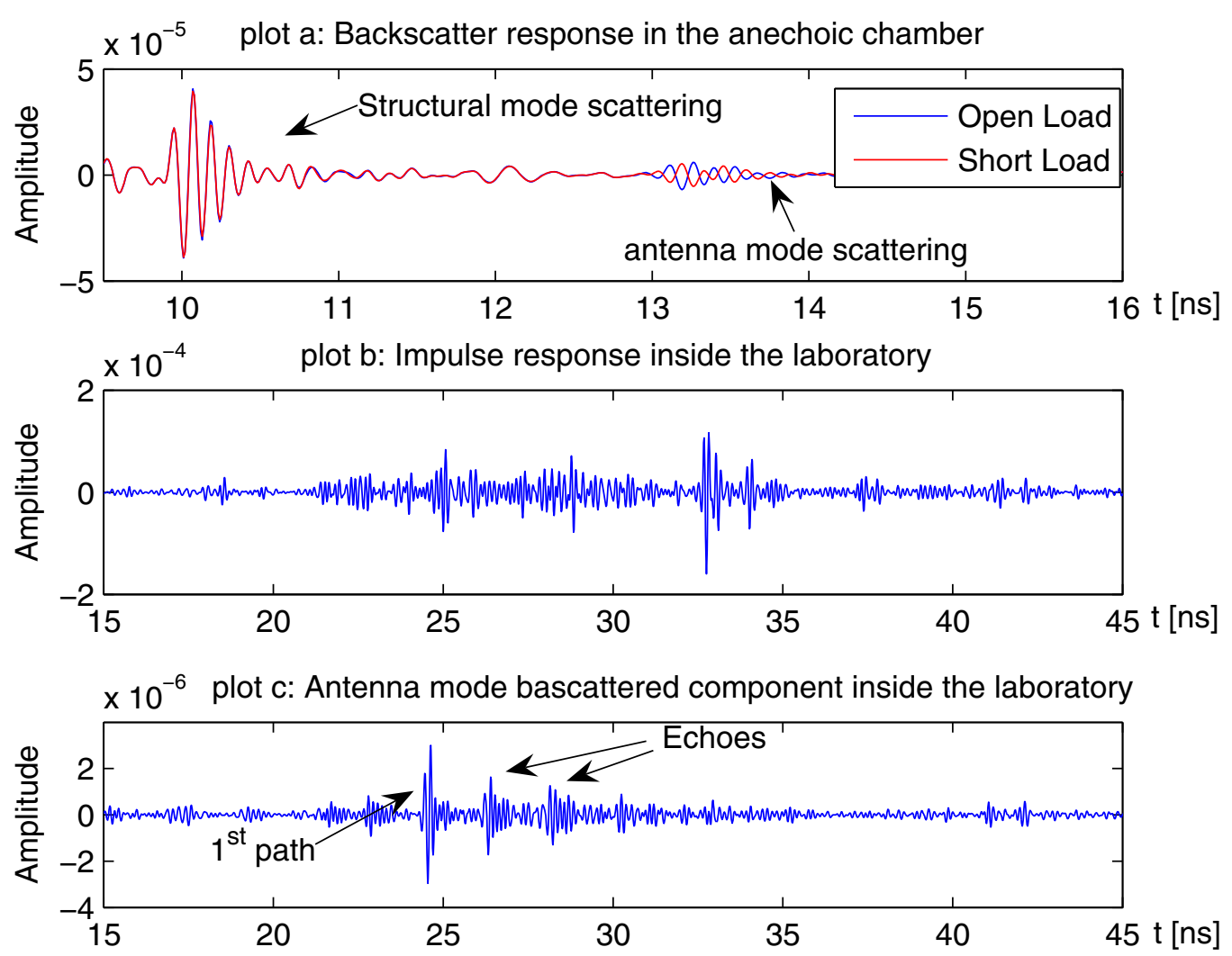

Figure 3 Example of backscatter responses collected inside in the anechoic chamber (plot a) at distance $d_{\text {ref }}=1.46 \mathrm{~m}$, in the laboratory (plot b) grid location $\mathrm{H}$ at distance of $3.10 \mathrm{~m}$, and of the only antenna mode contribution after clutter removal (plot c) in the same location $\mathrm{H}$.

to indirect paths between the tag and the reader. In most of the considered configurations, the normalized cross-correlation $\rho$ between the backscattered signals in the case of open- and short-circuit loads is close to -1 , as expected for antenna mode signals. This good symmetry property is useful in case of signaling schemes employing antipodal pulses and justifies, in the following analysis, the approximation of perfect pulse symmetry, i. e., $w(t)=w(t ; d, 0, \boldsymbol{\Theta})=-w(t ; d, 1, \boldsymbol{\Theta})$, where for notation compactness, we have hidden in $w(t)$ the explicit dependence on $d$ and $\boldsymbol{\Theta}$.

\section{Backscatter Communication Using UWB Signals}

\section{A. System model}

Consider a scenario where a reader interrogates $N_{\text {tag }}$ tags located in the same area. In Figure 4, the architectures for tag and reader are shown. The reader is composed of a transmitter and a receiver section both connected to the same UWB antenna through a TX/RX switch. During the interrogation phase, the reader transmits a sequence of UWB pulses, each having energy $E_{\mathrm{t}}$, modulated by a periodic binary sequence $\left\{a_{n}\right\}$ of period
$N_{s}$, with $a_{n} \in\{-1,+1\}$, specific to that particular reader (reader's code). Without loss of generality, an infinite interrogation sequence of pulses separated by $T_{\mathrm{f}}$ seconds (frame time) is considered, that is,

$$
s_{\text {reader }}(t)=\sum_{n=-\infty}^{\infty} a_{n} \cdot g\left(t-n T_{\mathrm{f}}\right) .
$$

The frame time $T_{\mathrm{f}}$ is chosen so that all backscattered signals are received by the reader before the transmission of the subsequent pulse, thus avoiding inter-frame interference. In indoor scenario, $T_{\mathrm{f}}=50-100 \mathrm{~ns}$ is usually sufficient to this purpose [38].

During the transmission of each pulse, the antenna is connected to the transmitter section. It is then kept connected to the receiver section during the remaining time until the following pulse is transmitted. Each pulse in (8) is backscattered by all tags as well as by all the surrounding scatterers present in the environment that form the clutter component.

The main task of the receiver section of the reader is to detect the useful backscattered signal component (i.e., 


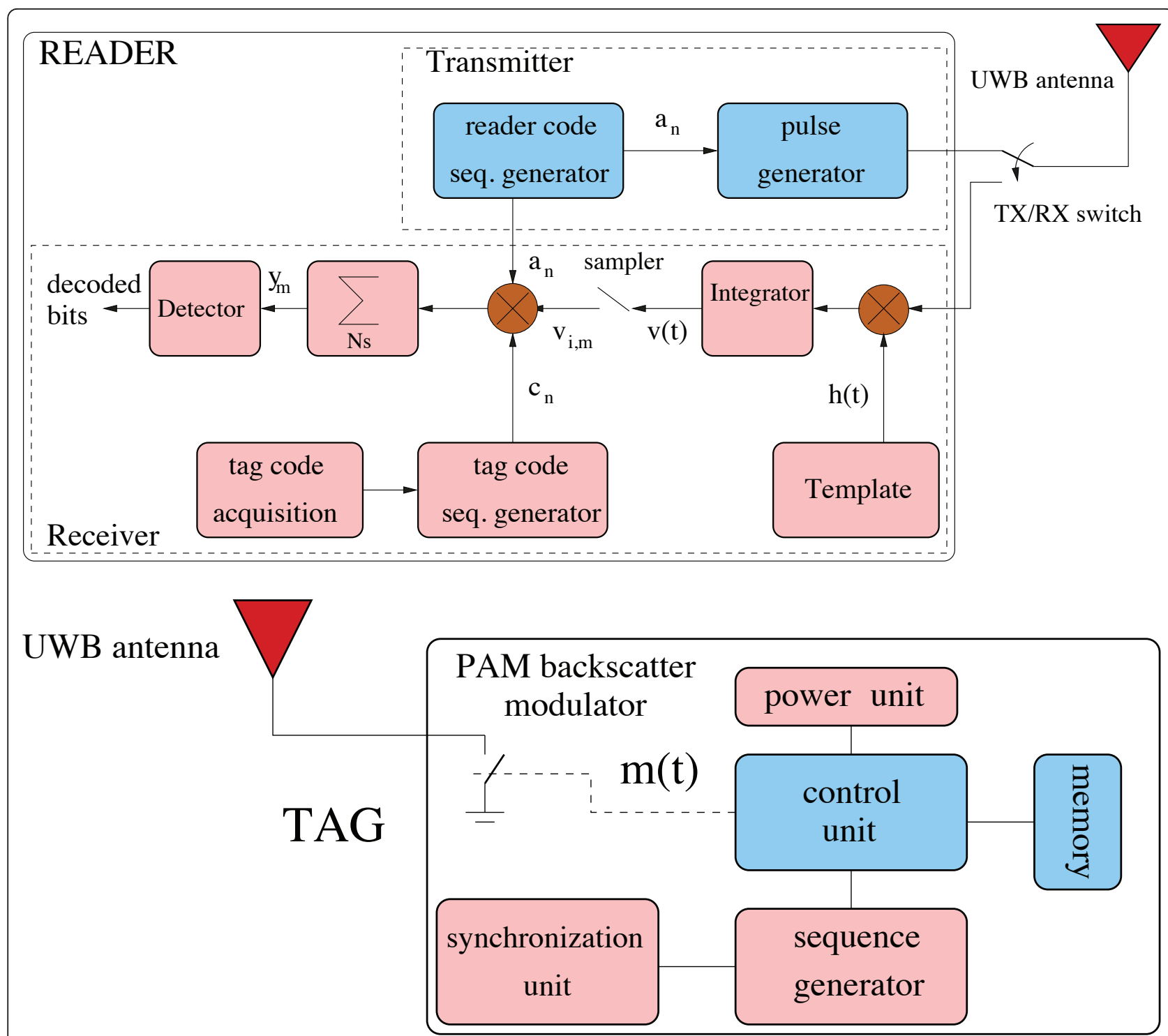

Figure 4 The considered scheme of the tag and the reader composed of a transmitter and a receiver section.

the antenna mode scattering dependent on antenna load changes) from the signals backscattered by the antenna structural mode and other scatterers (clutter), which are, in general, dominant. To this purpose, a quite general backscatter modulator architecture has been proposed in [24], allowing for different signaling schemes, such as pulse amplitude modulation (PAM), pulse position modulation (PPM), and ON-OFF keying. In this study, the performance of a simplified version of the tag architecture in [24] by considering the 2-PAM signaling is analyzed. In this case, the backscatter modulator reduces to a simple switch as shown in Figure 4. The analysis can be easily extended to other signaling schemes.

The reader and the tags have their own independent clock sources and hence they have to be treated as asynchronous. Let us denote $\Delta^{(k)}=\delta^{(k)}+T_{\mathrm{f}} u^{(k)}$, with $u^{(k)}$ integer and $0 \leq \delta^{(k)}<T_{\mathrm{f}}$, the clock offset of the $k$ th tag with respect to the reader's clock.

To make the uplink communication between the $k$ th tag and the reader robust to the presence of clutter, interference, and to allow multiple access, each tag is designed to change its status (short or open circuit) at each frame time $T_{\mathrm{f}}$ according to the data to be transmitted and a periodic tag's code $\left\{c_{n}^{(k)}\right\}$, with $c_{n}^{(k)} \in\{-1,+1\}$, of period $N_{\mathrm{s}}$. Specifically, each tag information symbol $d_{n}^{(k)} \in\{-1,+1\}$ is associated to $N_{\mathrm{s}}$ pulses, thus the symbol duration equals $T_{\mathrm{s}}=T_{\mathrm{f}} N_{\mathrm{s}}$. In this way, the polarity of the reflected signal changes according to the tag's code sequence during a symbol 
time, whereas the information symbol affects the entire sequence pulse's polarity at each symbol. Therefore, the backscatter modulator signal, commanding the tag's switch, can be expressed as

$$
\begin{aligned}
m^{(k)}(t) & =\sum_{n=-\infty}^{\infty} \sum_{i=0}^{N_{\mathrm{s}}-1} c_{i}^{(k)} d_{n}^{(k)} \Pi\left(\frac{1}{T_{\mathrm{f}}}\left[t-n T_{\mathrm{s}}-i T_{\mathrm{f}}-\Delta^{(k)}\right]\right) \\
& =\sum_{n=-\infty}^{\infty} c_{n}^{(k)} d_{f(n)}^{(k)} \Pi\left(\frac{1}{T_{\mathrm{f}}}\left[t-\left(n+u^{(k)}\right) T_{\mathrm{f}}-\delta^{(k)}\right]\right)
\end{aligned}
$$

having defined $f(n) \triangleq\left\lceil n / N_{\mathrm{s}}\right\rceil$ and $\Pi(t) \triangleq 1$ for $t \in[0,1]$ and zero otherwise. ${ }^{\mathrm{b}}$

In the following analysis, the tag response due to the antenna mode is examined whereas the antenna structural mode will be treated as part of the clutter, since it does not depend on data symbols. As a consequence, any clutter removal technique adopted will be also effective on the antenna structural mode component.

The signal received by the $k$ th tag is given by

$$
r_{\text {tag }}^{(k)}(t)=\sum_{n=-\infty}^{\infty} a_{n} \cdot p^{(k)}\left(t-n T_{\mathrm{f}}\right)
$$

where $p^{(k)}(t)$ is the downlink (reader-tag) channel response to $g(t)$ which includes also the propagation delay.

According to (10) and (9), and considering perfect pulse symmetry in the two antenna load conditions, the signal scattered by the $k$ th tag can be written as (see also the example in Figure 5)

$$
\begin{aligned}
& s_{\text {aga }}^{(k)}(t)=r_{\text {tag }}^{(k)}(t) \cdot m^{(k)}(t)
\end{aligned}
$$

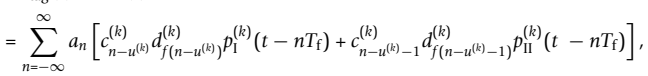

where we defined

$$
\begin{aligned}
& p_{\mathrm{I}}^{(k)}(t) \triangleq p^{(k)}(t) \cdot \Pi\left(\frac{t-\delta^{(k)}}{T_{\mathrm{f}}-\delta(k)}\right), \\
& p_{\mathrm{II}}^{(k)}(t) \triangleq p^{(k)}(t) \cdot \Pi\left(\frac{t}{\delta(k)}\right) .
\end{aligned}
$$

In (11), we assumed a perfect switch, i.e., a switch characterized by an instantaneous switching time as well as absence of ringing effects. These effects may not be negligible during the synchronization phase when load changes could happen in the middle of a pulse thus leading to pulse distortion. However, they do not affect the system performance once the reader has been synchronized to the backscattered signal. Synchronization techniques are investigated in [39] and are out of the scope of this article.
The received signal at the reader is ${ }^{\mathrm{c}}$

$$
r_{\text {reader }}(t)=\sum_{k=1}^{N_{\text {tag }}} r_{\text {reader }}^{(k)}(t)+\sum_{n=-\infty}^{\infty} a_{n} w^{(\mathrm{C})}\left(t-n T_{\mathrm{f}}\right)+n(t),
$$

where $n(t)$ is the additive white Gaussian noise (AWGN) with two-sided power spectra density $N_{0} / 2$ and $w^{(\mathrm{C})}(t)$ is the backscattered version of the pulse $g(t)$ due to the clutter component which also accounts for pulse distortion, multipath propagation, and tag's antenna structural mode. The signal $r_{\text {reader }}^{(k)}(t)$ represents the received useful component due to the $k$ th tag, i.e.,

$$
\left.r_{\text {reader }}^{(k)}(t)=\sum_{n=-\infty}^{\infty} a_{n}\left[c_{n-u^{(k)}}^{(k)} d_{f\left(n-u^{(k)}\right)}^{(k)} w_{1}^{(k)}\left(t-n T_{\mathrm{f}}\right)+c_{\left.n-u^{(k)}\right)-1}^{(k)} d_{f\left(n-u^{(k)}-1\right)}^{(k)}\right)_{11}^{(k)}\left(t-n T_{\mathrm{f}}\right)\right]
$$

having denoted $w_{\mathrm{I}}^{(k)}(t)$ and $w_{\mathrm{II}}^{(k)}(t)$, respectively, for the uplink channel response to $p_{\mathrm{I}}^{(k)}(t)$ and $p_{\mathrm{II}}^{(k)}(t)$ (see Figure 5). Note that $w^{(k)}(t)=w_{\mathrm{I}}^{(k)}(t)+w_{\mathrm{II}}^{(k)}(t)$ is the round-trip response to $g(t)$ of the backscatter link defined in Section 2.

Consider the reader's receiver scheme reported in Figure 4 where the received signal is correlated with a local waveform template $h(t)$ with unitary energy. The output is then sampled at intervals $t_{i, m}=i T_{\mathrm{f}}+m T_{\mathrm{s}}+\tau_{0}$, with $i=0,1, \ldots, N_{\mathrm{s}}-1$, thus obtaining the following samples:

$$
\left.v_{i, m}=\int_{0}^{T_{\mathrm{f}}} h(t)\right)_{\text {reader }}\left(t-t_{i, m}\right) d t=Y_{\text {reader }}\left(t_{i, m}\right) \otimes h\left(-t_{i, m}\right)=\sum_{k=1}^{N_{\text {nag }}} v_{i, m}^{(k)}+v_{i, m}^{(\mathrm{C})}+z_{i, m}
$$

where $^{\mathrm{d}}$

$$
\begin{aligned}
& v_{i, m}^{(k)}=\sum_{n=-\infty}^{\infty} a_{n}\left[c_{n-u^{(k)}}^{(k)} d_{f\left(n-u^{(k)}\right)}^{(k)} \gamma_{1}^{(k)}\left(i T_{\mathrm{f}}+m T_{\mathrm{s}}+\tau_{0}-n T_{\mathrm{f}}\right)\right. \\
&\left.+c_{n-u^{(k)}-1}^{(k)} d_{f\left(n-u^{(k)}-1\right)}^{(k)} \gamma_{\mathrm{II}}^{(k)}\left(i T_{\mathrm{f}}+m T_{\mathrm{s}}+\tau_{0}-n T_{\mathrm{f}}\right)\right], \\
& v_{i, m}^{(C)}=\sum_{n=-\infty}^{\infty} a_{n} \gamma^{(C)}\left(i T_{\mathrm{f}}+m T_{\mathrm{s}}+\tau_{0}-n T_{\mathrm{f}}\right) .
\end{aligned}
$$

In (16) and (17), we have defined $\gamma_{\mathrm{II}}^{(k)}(t) \triangleq w_{\mathrm{II}}^{(k)}(t) \otimes h(-t), \gamma_{\mathrm{II}}^{(k)}(t) \triangleq w_{\mathrm{II}}^{(k)}(t) \otimes h(-t), \quad \gamma^{(\mathrm{C})}$ $(t) \triangleq w^{(\mathrm{C})}(t) \otimes h(-t), z(t) \triangleq n(t) \otimes h(-t)$, and $z_{i, m} \triangleq z\left(i T_{\mathrm{f}}+\right.$ $\left.m T_{\mathrm{s}}+\tau_{0}\right)$. $^{\mathrm{e}}$

Without loss of generality, we consider the problem of detecting the data bit $d_{m}^{(1)}$ of tag \#1 (useful tag). As shown in Figure 4, to remove the clutter component at the receiver, the sampled signal $v_{i, m}$ is multiplied by the composite sequence $\left\{c_{n}^{(1)} \cdot a_{n}\right\}$, which identifies both the reader and the desired tag \#1. ${ }^{\mathrm{f}}$ In particular, all the resulting $N_{\mathrm{s}}$ samples at the output of the correlator composing a data symbol are summed up to form the $m$ th decision variable at the detector input. Considering 


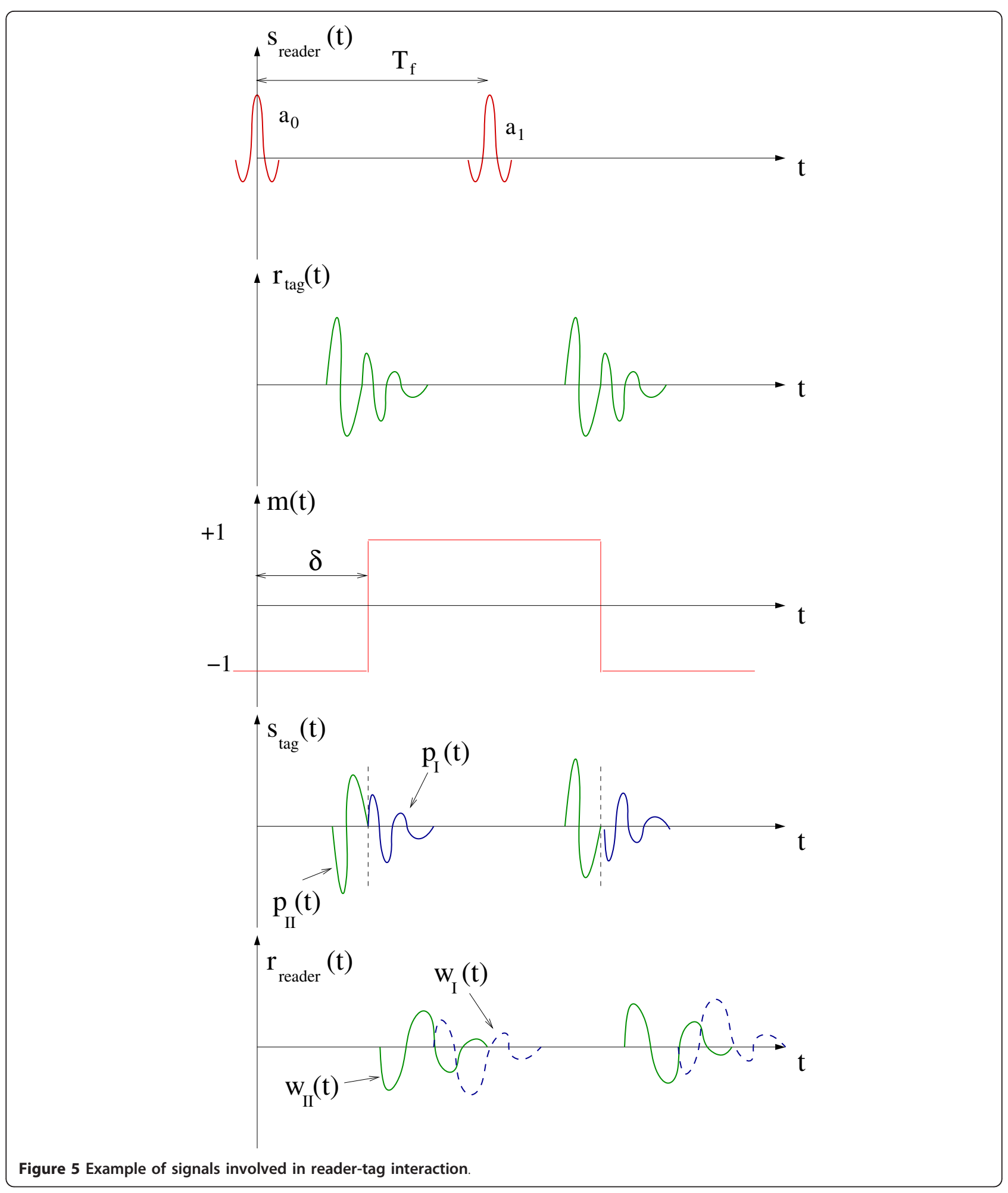


that $c_{i+m N_{\mathrm{s}}}^{(k)}=c_{i}^{(k)}$, and $a_{i+m} N_{s}=a_{i} \forall i$, the decision variable for the $m$ th symbol $d_{m}^{(1)}$ becomes

$$
\begin{aligned}
\gamma_{m}= & \sum_{i=0}^{N_{s}-1} c_{i}^{(1)} a_{i} v_{i, m} \\
= & \gamma_{\mathrm{I}}^{(1)}\left(\tau_{0}\right) \sum_{i=0}^{N_{s}-1}\left[a_{i}^{2} c_{i}^{(1)} c_{i-u^{(1)}}^{(1)} d_{f\left(m-u^{(1)}\right)}^{(1)}\right]+\gamma_{I I}^{(1)}\left(\tau_{0}\right) a_{0}^{2} c_{0}^{(1)} c_{-u^{(1)}-1}^{(1)} d_{f\left(m-u^{(1)}-1\right)}^{(1)} \\
& +\gamma_{\text {II }}^{(1)}\left(\tau_{0}\right) \sum_{i=1}^{N_{s}-1}\left[a_{i}^{2} c_{i}^{(1)} c_{i-u^{(1)}-1}^{(1)} d_{f\left(m-u^{(1)}-1\right)}^{(1)}\right]+\xi_{m}+\gamma_{m}^{(C)}+z_{m}
\end{aligned}
$$

where

$$
\gamma_{m}^{(C)}=\sum_{i=0}^{N_{\mathrm{s}}-1} c_{i}^{(1)} a_{i} \sum_{n=-\infty}^{\infty} a_{n} \gamma^{(C)}\left(i T_{\mathrm{f}}+m T_{\mathrm{s}}+\tau_{0}-n T_{\mathrm{f}}\right)=\gamma^{(C)}\left(\tau_{0}\right) \sum_{i=0}^{N_{\mathrm{s}}-1} c_{i}^{(1)}
$$

and $z_{m} \triangleq \sum_{i=0}^{N_{\mathrm{s}}-1} a_{i} c_{i} z_{i, m}$ is a Gaussian distributed random variable (RV) with zero mean and variance $\sigma_{z}^{2}=N_{\mathrm{s}} N_{0} / 2$

The component $\xi_{m}$ accounts for the MUI and can be expressed as follows:

$$
\begin{aligned}
\xi_{m}= & \sum_{k=2}^{N_{\text {tag }}} \sum_{i=0}^{N_{s}-1} c_{i}^{(1)} a_{i} v_{i, m}^{(k)} \\
= & \sum_{k=2}^{N_{\text {tag }}}\left\{\gamma_{1}^{(k)}\left(\tau_{0}\right) \sum_{i=0}^{N_{s}-1}\left[a_{i}^{2} c_{i}^{(1)} c_{i-u^{(k)}}^{(k)} d_{f\left(m-u^{(k)}\right)}^{(k)}\right]+\gamma_{\mathrm{II}}^{(k)}\left(\tau_{0}\right) a_{0}^{2} c_{0}^{(1)} c_{-u^{(k)}-1}^{(k)} d_{f\left(m-u^{(k)}-1\right)}^{(k)}\right. \\
& \left.+\gamma_{\mathrm{II}}^{(k)}\left(\tau_{0}\right) \sum_{i=1}^{N_{s}-1}\left[a_{i}^{2} c_{i}^{(1)} c_{i-u^{(k)}-1}^{(k)} d_{f\left(m-u^{(k)}-1\right)}^{(k)}\right]\right\}
\end{aligned}
$$

whose effect on the decision variable strictly depends on the cross-correlation property between codes $\left\{c_{i}^{(1)}\right\}$ and $\left\{c_{i}^{(k)}\right\}$.

In the following, we assume that code synchronization is achieved after an initial acquisition phase, i.e., $u^{(1)}=$ 0 . To this purpose, powerful acquisition techniques (e.g., [40]) can be adopted. From (18), we have

$$
\gamma_{m}=d_{m}^{(1)}\left[\gamma_{1}^{(1)}\left(\tau_{0}\right) N_{s}+\gamma_{\Pi}^{(1)}\left(\tau_{0}\right) \sum_{i=1}^{N_{s}-1} c_{i-1}^{(1)} c_{i}^{(1)}\right]+\gamma_{\Pi}^{(1)}\left(\tau_{0}\right) c_{-1}^{(1)} c_{0}^{(1)} d_{m-1}^{(1)}+\xi_{m}+\gamma_{m}^{(C)}+z_{m} .
$$

Looking at (21), it can be noted that the useful term depends on the partial autocorrelation properties of code $\left\{c_{i}^{(1)}\right\}$.

As a further hypothesis, we assume that a perfect TOA estimate is available. The TOA estimator robust to clutter proposed in [39] can be adopted to this purpose. Once the TOA is known, the reader can adjust its internal clock so that it becomes synchronous to that of the intended tag, i.e., $\delta^{(1)}=0$, and the optimal choice for $\tau_{0}$ can be derived. In such a case ${ }^{g}$

$$
\gamma_{\mathrm{I}}^{(1)}\left(\tau_{0}\right)=E_{\mathrm{W}}=\int_{-\infty}^{\infty}\left(w^{(1)}(t)\right)^{2} d t
$$

and (21) can be further simplified leading to

$$
y_{m}=d_{m}^{(1)} N_{\mathrm{s}} E_{\mathrm{w}}+\xi_{m}+\gamma_{m}^{(C)}+z_{m}=d_{m}^{(1)} \rho E_{\mathrm{s}}+\gamma_{m}^{(C)}+\xi_{m}+z_{m},
$$

where $E_{\mathrm{s}}=N_{\mathrm{s}} E_{\mathrm{w}}$, and $\rho$ is the normalized cross-correlation between pulses $w_{\mathrm{I}}^{(1)}(t)$ and $h(t)$, which accounts for the mismatch due to pulse distortion. Parameters $E_{\mathrm{w}}$ and $E_{\mathrm{s}}$ represent the average received energy per pulse and symbol, respectively. ${ }^{\mathrm{h}}$ For further convenience, we define the signal-to-clutter ratio $(\mathrm{SCR})$ as $\mathrm{SCR}=\frac{E_{\mathrm{s}}}{N_{\mathrm{s}} E_{\mathrm{c}}}$, where $E_{\mathrm{C}}=\int_{0}^{T_{\mathrm{f}}}\left(w^{(C)}(t)\right)^{2} d t$ is the energy per pulse of the clutter component.

Note that the accurate estimation of $\tau_{0}$, which is a peculiarity of UWB signals, allows for high accuracy ranging, and hence high accuracy localization of the tag when at least three readers access the tag $[12,15,41]$.

\section{B. Code choice for clutter removal and multiple access}

Looking at (18) and (19), it can be noted that only the antenna mode-scattered signals are modulated by the combination of the tag's and reader's codes $\left\{c_{i}^{(k)}\right\}$ and $\left\{a_{i}\right\}$, whereas all the clutter signals' components (including the antenna structural mode scattering) are received modulated only by the reader's code $\left\{a_{i}\right\}$. This suggests that, as can be deduced from (19), to completely remove the clutter component, and hence the antenna structural mode component, it is sufficient that the tag's code $\left\{c_{i}^{(1)}\right\}$ has zero mean, i.e., $\sum_{n=0}^{N_{\mathrm{s}}-1} c_{n}^{(1)}=0$, leading to $y_{m}^{(C)}=0$, if a quasi-stationary scenario within the symbol time $T_{\mathrm{s}}$ is assumed.

Regarding the MUI, the situation is similar to what happens in conventional code division multiple access systems where the performance is strictly related to the partial cross-correlation properties of codes $\left\{c_{i}^{(1)}\right\}$ and $\left\{c_{i}^{(k)}\right\}[42]$. Classical codes such as Gold codes or $m$ sequences offer good performance. Unfortunately, they are composed of an odd number of symbols, and hence there is no way to obtain a zero mean code to completely remove the clutter. However, considering that $m$ sequences have a quasi-balanced number of " +1 " and "-1," i.e., their number differs no more than 1, one option to achieve clutter removal is to lengthen the code by one symbol so that the resulting code had a zero mean. As a consequence, we expect a certain degradation in terms of multiple access performance, especially when short codes are adopted. In the numerical results, this aspect will be investigated.

When the scenario is quasi-synchronous, i.e., $u^{(k)}=0$ $\forall k$ and $\delta^{(k)} \neq 0$, orthogonal codes, such as Hadamard codes, represent a good choice [43] and $\xi_{m}=0$. This could be the situation where a wake up signal is sent by the reader to switch the tag on and reset the code phase. Such a solution is under investigation in [27], 
where the UWB tag is supposed to be woken up by a dedicated control signal sent in the UHF band.

\section{Performance in a single-tag scenario}

We now investigate the performance in a single-tag scenario to gain some insights about the attainable ultimate performance using the backscatter communication mechanism. The complete multi-tag scenario will be investigated in the numerical results using experimental data. In the absence of other tags in the environment, we have $\xi_{m}=0$, whereas clutter contribution is completely suppressed thanks to the adoption of zero mean codes.

The simplest UWB receiver is the single-path matched filter (SPMF) [44] where, in the absence of other information, $h(t)$ can be chosen to be proportional to $w(t$; $\left.d_{\text {ref }}, 0, \boldsymbol{\Theta}_{\max }\right)$, i.e., the received pulse at the reference distance $d_{\text {ref }}$ in free-space propagation at the orientation $\boldsymbol{\Theta}_{\max }$ of maximum tag's antenna radiation. This receiver is optimal in AWGN at $\boldsymbol{\Theta}_{\max }$, but it is in general suboptimal in a multipath scenario. In such a case, the receiver in Figure 4 can easily be extended to obtain a Rake structure composed of a number $L_{\mathrm{p}}$ of fingers, each of them synchronized to a different path. The performance of the SPMF, or any other receiver solutions such as those based on Rake structures, are bounded by the ideal matched filter (IMF) receiver. The IMF receiver is equivalent to a Rake receiver with unlimited correlators (all Rake Receiver) and a perfect estimate of the received waveform to be used as local template $h(t)$ $[44,45]$. This means that $h(t)$ is proportional to the effective received waveform $w(t)$ and, consequently, the performance of the IMF can be obtained by setting $\rho=$ 1 in (23). In general, from (23), it is easy to show that the bit error probability (BEP) is given simply by

$$
P_{\mathrm{b}}=\frac{1}{2} \operatorname{erfc}\left(\sqrt{\frac{E_{\mathrm{s}} \rho^{2}}{N_{0}}}\right),
$$

where $\operatorname{erfc}(\cdot)$ is the complementary error function.

For further convenience, we define $G_{\text {ref }}=E_{\text {ref }} / E_{\mathrm{t}}$, i.e., the round-trip channel power gain at the reference distance $d_{\text {ref }}$ and at the maximum direction of radiation $\boldsymbol{\Theta}_{\max }$ in AWGN scenario, where $E_{\text {ref }}$ is the received energy per pulse at the reference distance $d_{\text {ref }}$. In addition, we assume a typical exponential path loss law where the power path loss exponent $\beta$ usually ranges between $\approx 1.8$ and $\approx 4$ [38]. The BEP can be rewritten as

$$
P_{\mathrm{b}}=\frac{1}{2} \operatorname{erfc}\left(\sqrt{\frac{P_{\mathrm{t}} G_{\mathrm{ref}} \rho^{2}\left(\frac{d_{\mathrm{ref}}}{d}\right)^{2 \beta}}{N_{0} R_{\mathrm{b}}}}\right),
$$

where $R_{\mathrm{b}}=1 /\left(N_{\mathrm{s}} T_{\mathrm{f}}\right)$ is the data rate (symbol rate), and $P_{\mathrm{t}}=\frac{E_{\mathrm{t}}}{T_{\mathrm{f}}}$. It is interesting to note that the exponent $2 \beta$ is present in (25) instead of $\beta$ to account for the two-way link.

\section{Numerical Results}

In order to evaluate the performance of the proposed passive UWB RFID communication system, the following parameter values have been considered: $T_{\mathrm{f}}=100 \mathrm{~ns}$, $F=4 \mathrm{~dB}$ (receiver noise figure), and effective radiated isotropic power (EIRP) EIRP $=-6.7 \mathrm{dBm}$.

\section{A. Range-data rate trade-off in single-tag anechoic chamber scenario}

Figure 6 shows the achievable operating range as a function of the data rate $R_{\mathrm{b}}$ for a fixed target BEP $P_{\mathrm{b}}=10^{-3}$. A SPMF receiver in anechoic chamber scenario (AWGN channel) is considered using measurement data for different tag's antenna orientation offsets $\varphi$ with respect to the maximum radiating angle. For each measured dataset, the normalized cross-correlation coefficient $\rho$ was calculated and used in (25). For the BAV antenna, $G_{\text {ref }}$ $=-75 \mathrm{~dB}$, which accounts also for the reader's antenna gain $G_{\text {reader }}=5 \mathrm{~dB}$. From the figure, it can be seen that, for example, for data rate $R_{\mathrm{b}}=10^{3} \mathrm{bits} / \mathrm{s}$, an operating range larger than $20 \mathrm{~m}$ can be achieved. However, antenna radiation pattern and pulse distortion might determine a significant performance degradation when devices are not oriented to the maximum radiating direction as can be noted in Figure 6 (see curves with $\varphi$

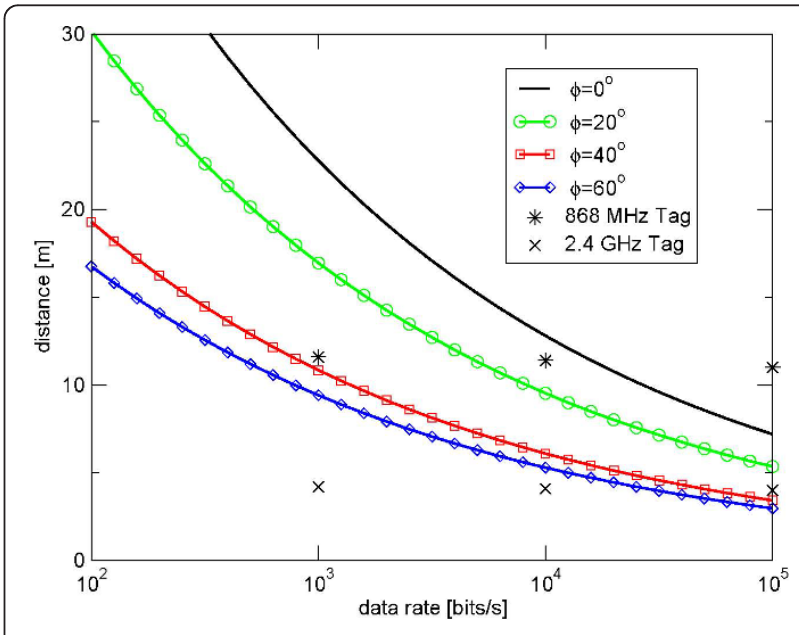

Figure 6 Tag-reader operating range in meters as a function of the data rate for $P_{\mathrm{b}}=10^{-3}$ and different tag orientations in the anechoic chamber scenario. BAV antenna for the tag is considered. Cross and star dots refer to the corresponding performance of UHF tags operating at $868 \mathrm{MHz}$ and $2.4 \mathrm{GHz}$, respectively (from [19]). 
$\neq 0$ ). This degradation can be mitigated using, for example, multiple readers or antennas [46]. For comparison, the corresponding operating ranges in free-space conditions are reported for UHF-passive RFID tags operating at $868 \mathrm{MHz}$ and $2.4 \mathrm{GHz}$, respectively, with a transmitted EIRP of $500 \mathrm{~mW}$ according to European regulations [19]. As can be noted, using the UWB technology a significantly larger operating range is achievable with respect to that of UHF-based RFID systems, especially for low data rates, with a dramatically reduced transmitted power level $(\approx 0.09$ vs. $500 \mathrm{~mW})$. It is important to remark that the ongoing study in the European Regulatory Framework is trying to establish that location tracking equipment, operating indoors in the reduced frequency band 6.4-8.5 GHz, could increase both the average and peak effective isotropically radiated powers by up to $10 \mathrm{~dB}(-31.3$ and $10 \mathrm{dBm} / 50 \mathrm{MHz}$, respectively), provided their duty cycle does not exceed $2.5 \%$ [20]. This would mean 10 times increased data rate, thus making passive UWB RFID very attractive for next generation RFID systems as complementary or integrating technology [27].

\section{B. BER-SIR in multi-tags anechoic chamber scenario with artificial clutter}

We evaluate the BER in the presence of an interfering tag as a function of the signal-to-interference ratio (SIR). The signal-to-noise ratio (SNR) has been fixed to $7 \mathrm{~dB}$, corresponding to an error floor of about $10^{-3}$ in the absence of interference. Results have been obtained by Monte Carlo simulations starting from antenna backscatter measurements in anechoic chamber. The interfering tag, the clutter and the thermal noise signal components have been added artificially according to the set SNR, SIR, and SCR values. The clutter waveform has been taken from measurements in the laboratory environment. The worst-case scenario with the interfering signal completely overlapped to the useful one is considered.

In Figure 7, results associated to different spreading codes are compared. In the quasi-synchronous scenario, orthogonal Hadamard codes are used. As expected, the performance is not sensitive to the presence of both the interference and the clutter (because of the zero-mean code used). In the asynchronous scenario, $m$-sequences spreading codes of length 7 and 63 have been considered. From the curve corresponding to $m$-seq. 7 , it can be observed that when the clutter is present and it is significative ( $\mathrm{SCR}=-34 \mathrm{~dB}$ ), even for large SIR values, the performance is limited by the clutter. A remarkable improvement can be obtained by extending the length of the code by one (zero-mean code). When longer codes are used (e.g., $m$-seq. 63), the impact of clutter becomes less significant and good performance can be

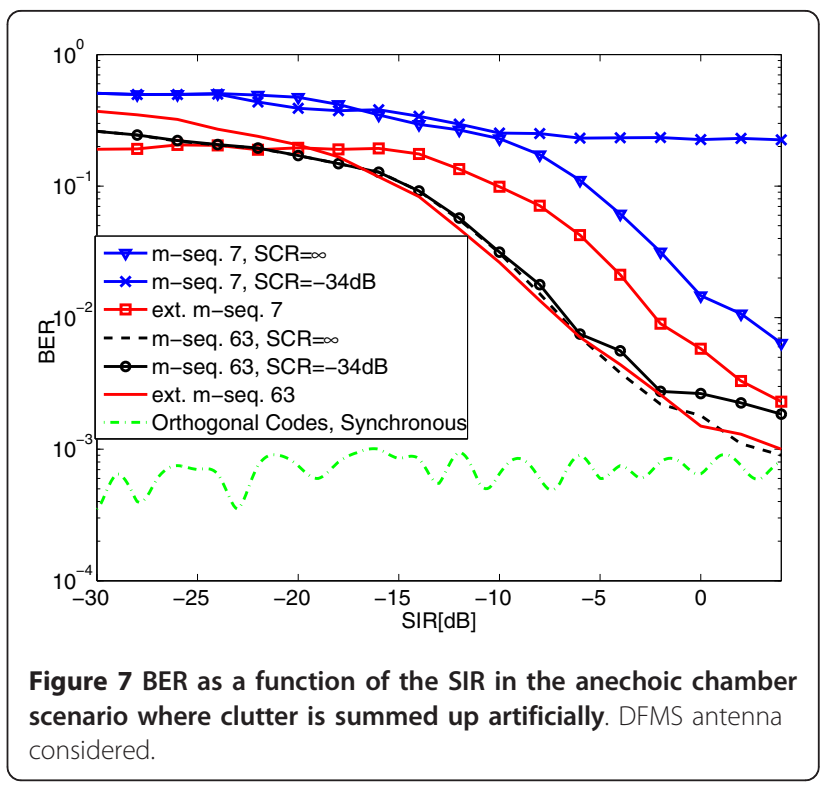

achieved even using quasi zero-mean codes. These considerations suggest that the adoption of extended $m$ sequences is the appropriate choice, especially when working with short codes.

\section{BEP-data rate in single-tag laboratory scenario}

Results related to the SPMF and IMF receivers in every location in the grid inside the laboratory are shown in Figures 8 and 9 , respectively, in terms of BEP calculated using (25) and deriving $\rho$ from measurements. For the sake of comparison, the performance in AWGN is also reported for $d=1.46 \mathrm{~m}$.

As expected, the performance of the IMF receiver is significantly better than that obtained using the simple SPMF receiver because all the useful energy coming

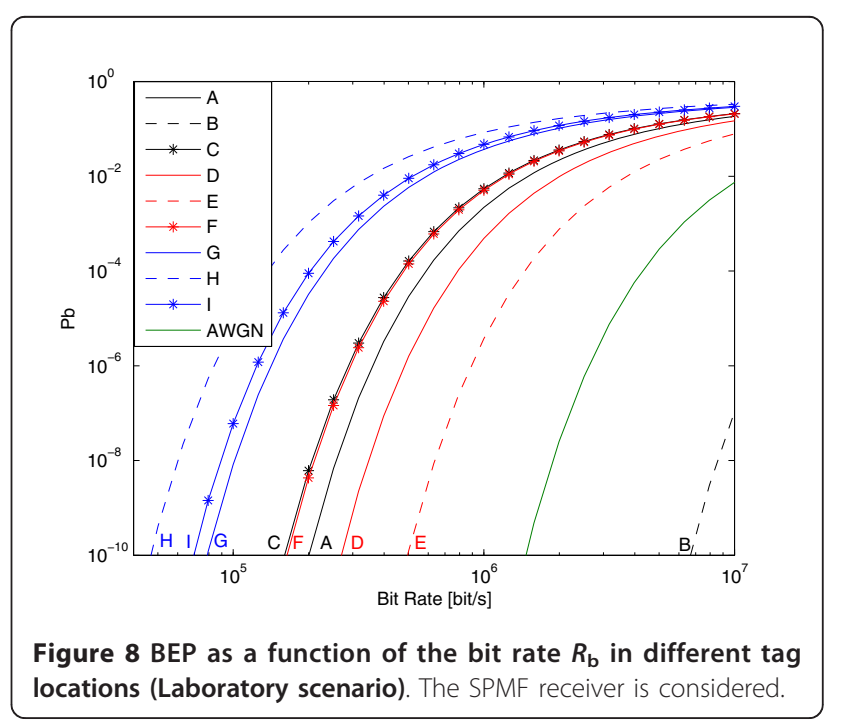




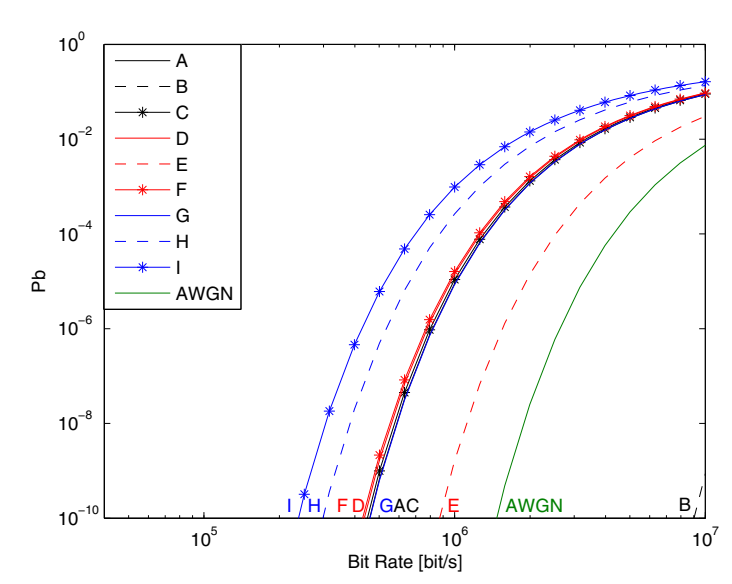

Figure 9 BEP as a function of the bit rate $R_{\mathrm{b}}$ in different tag locations (Laboratory scenario). The IMF receiver is considered.

from multipath is captured and the performance depends only on the received power. In fact, the SPMF receiver is not able to collect the energy from multipath and suffers from pulse distortion due to propagation as well as antenna effects. This problem can be mitigated by considering more complex receiver structures such as those based on Rake solutions performance of which is bounded by the one provided by the IMF receiver. The performance obtained with the tag located at location $B$ is better than that in AWGN condition because of the shorter distance $(1.10$ vs. $1.46 \mathrm{~m})$. Note that in some cases tags placed at larger distances provide a better performance. This depends on the higher amount of energy that can be collected in some locations because of the presence of richer multipath components in the received signal.

As a numerical example, with a target BEP $P_{\mathrm{b}}=10^{-3}$, data rates up to $200 \mathrm{kbit} / \mathrm{s}$ at a distance of $3.10 \mathrm{~m}$ with a transmitted power lower than $1 \mathrm{~mW}$ are feasible in a realistic environment.

\section{BER-SNR in multi-tag laboratory scenario}

In Figure 10, the bit error rate (BER) as a function of the SNR when $N_{\text {tag }}=6$ tags are present is reported. The signal measured from the location D of the grid is considered as the signal backscattered by the useful tag, and then we associate the backscattered signals coming from locations A, B, C, E, and F (see the map in Figure 2) to the interfering signals. Different SNR values have been obtained by adding the correspondent artificial thermal noise.

It is possible to see the performance gain obtained when the extended $m$-sequence 63 is adopted. This indicates that the effect of the clutter is dominant with respect to the effect of the MUI. A similar conclusion

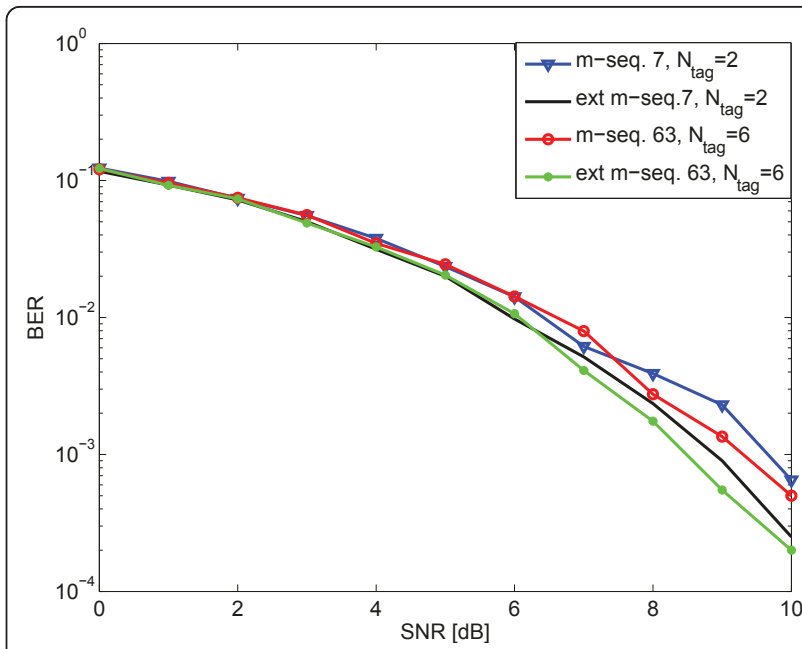

Figure 10 BER as a function of SNR (Laboratory scenario). The useful tag is located in point $D$. Five interfering tags are present in locations A, B, C, E, and F when $m$-sequence 63 is adopted. One interferer tag in location $F$ is considered when $m$-sequence 7 is used.

can be drawn when only one interfering tag located in $\mathrm{F}$ is present and an extended $m$-sequence 7 is considered.

\section{Conclusions}

UWB technology in next generation RFID systems is a promising solution to overcome most of the limitations of current narrow bandwidth RFID technology. In this article, we have addressed UWB RFID systems adopting backscatter modulation by proposing a reader-and-tag architecture capable of working in the presence of strong clutter and interference. The achievable range and performance has been investigated using measured data collected in controlled and realistic environments. It has been shown that clutter is the main limiting factor and that it can be mitigated or suppressed through the architecture proposed in this article and the adoption of zero mean spreading codes without compromising the performance in multi-tag scenario. Numerical results show that operating ranges of several meters are feasible in realistic scenarios with a transmitted power less than $1 \mathrm{~mW}$.

\section{Competing interests}

The authors declare that they have no competing interests.

\section{Endnotes}

${ }^{\text {a }}$ The structural mode-scattered component here is conventionally defined as the signal scattered when the antenna load is matched. In the literature, it is often defined with respect to the short-circuit load [28]. In 
any case, whatever convention is adopted, the following analysis and the results are not affected. ${ }^{\mathrm{b}}$ Operator $\lceil x\rceil$ denotes the smallest integer larger than or equal to $x$. ${ }^{\mathrm{c}}$ Coupling effects between close tags are not considered here. They deserve future investigations, even though we expect that, in most cases, their impact on system performance is negligible thanks to the different spreading codes adopted in each tag. ${ }^{\mathrm{d}} \otimes$ denotes the convolution operator. ${ }^{\mathrm{e}}$ According to the design criteria for $T_{\mathrm{f}}$ illustrated before, the support of $\gamma(t)$ belongs to $\left[0, T_{\mathrm{f}}\right]$, and no inter-frame interference is present. ${ }^{\mathrm{f}}$ Multiple readers may access the same tag by using different reader codes provided that they are designed with good cross-correlation properties. ${ }^{\mathrm{g}}$ Note that under perfecttiming condition, it is $w_{\mathrm{I}}^{(1)}(t)=w^{(1)}(t)$ and $w_{\mathrm{II}}^{(1)}(t)=0$. Moreover, as already mentioned, any non ideal switching effect becomes negligible because it affects parts of the received signal not interested by the useful backscattered pulse. ${ }^{\text {h}}$ Note that the energy of the useful tag response may vary for different delays and codes when the reader is not synchronized. In such a case, (22) does not hold anymore.

\section{Acknowledgements}

The authors would like to thank Raffaele D'Errico for his assistance on measurement equipment and Nicolò Decarli for his useful suggestions. This study has been performed within the framework FP7 European Project SELECT (Grant no. 257544).

\section{Author details}

${ }^{1}$ WiLAB, Dipartimento di Elettronica, Informatica e Sistemistica (DEIS), University of Bologna at Cesena, Via Venezia 52, Cesena (FC) 47521, Italy ${ }^{2}$ ENSTA-ParisTech 32 Boulevard Victor, Paris Cedex 15 75739, France

Received: 30 August 2010 Accepted: 28 July 2011

Published: 28 July 2011

\section{References}

1. K Finkenzeller, RFID Handbook: Fundamentals and Applications in Contactless Smart Cards and Identification, 2nd edn. (Wiley, 2004)

2. $V$ Chawla, DS Ha, An overview of passive RFID. IEEE Appl Pract. 45, 11-17 (2007)

3. European Commission DG Information Society and Media/Unit G2 "Microsystems". To strengthen european technology: the support of RFID within FP7 (September 2007)

4. EPCGlobal, Class 1 generation 2 UHF air interface protocol standard v.1.0.9 (2005)

5. E Ngai, KK Moon, FJ Riggins, CY Yi, RFID research: an academic literature review (1995-2005) and future research directions. Int J Prod Econ. 112(2) 510-520 (2008). special Section on RFID: Technology, Applications, and Impact on Business Operations. doi:10.1016/j.jpe.2007.05.004

6. R Verdone, D Dardari, G Mazzini, A Conti, Wireless Sensor and Actuator Networks: Technologies, Analysis and Design (Elsevier Ltd, London, 2008)

7. D Kim, M Ingram, WJ Smith, Measurements of small-scale fading and path loss for long range RF tags. IEEE Trans Antennas Propag 51(8), 1740-1749 (2003). doi:10.1109/TAP.2003.814752

8. D Ha, P Schaumont, Replacing cryptography with ultra wideband (UWB) modulation in secure RFID, in 2007 IEEE International Conference on RFID, Grapevine, TX, USA (March 2007)

9. C Mutti, C Floerkemeier, in CDMA-based RFID systems in dense scenarios: concepts and challenges, Las Vegas, USA 215-222 (April 2008)
10. T van Deursen, S Radomirović, Security of RFID protocols-a case study. Electron Notes Theor Comput Sci. 244, 41-52 (2009)

11. J Griffin, G Durgin, Reduced fading for RFID tags with multiple antennas, in Antennas and Propagation International Symposium, 2007 IEEE, pp. 1201-1204 (June 2007)

12. D Dardari, R D'Errico, C Roblin, A Sibille, MZ Win, Ultrawide bandwidth RFID: the next generation? Proc IEEE. 98(9), 1570-1582 (2010) (Special Issue on RFID-A Unique Radio Innovation for the 21st Century)

13. MZ Win, RA Scholtz, Impulse radio: how it works. IEEE Commun Lett. 2(2), 36-38 (1998). doi:10.1109/4234.660796

14. MZ Win, D Dardari, AF Molisch, W Wiesbeck, J Zhang, History and Applications of UWB. Proc IEEE. 97(2), 198-204 (2009) (Special Issue on Ultra-Wide Bandwidth (UWB) Technology \& Emerging Applications)

15. D Dardari, A Conti, U Ferner, A Giorgetti, MZ Win, Ranging with ultrawide bandwidth signals in multipath environments. Proc IEEE. 97(2), 404-426 (2009) (Special Issue on UWB Technology \& Emerging Applications)

16. A Giorgetti, M Chiani, MZ Win, The effect of narrowband interference on wideband wireless communication systems. IEEE Trans Commun. 53(12), 2139-2149 (2005). doi:10.1109/TCOMM.2005.860047

17. RJ Fontana, SJ Gunderson, Ultra-wideband precision asset location system, in Proc of IEEE Conf on Ultra Wideband Systems and Technologies (UWBST). 21(1), 147-150 (May 2002)

18. D Dardari, Pseudo-random active UWB reflectors for accurate ranging. IEEE Commun Lett. 8(10), 608-610 (2004). doi:10.1109/LCOMM.2004.836838

19. GD Vita, G lannaccone, Design criteria for the RF section of UHF and microwave passive RFID transponders. IEEE Trans Microwave Theory Tech. 59(9), 2978-2990 (2005)

20. W Hirt, The european UWB radio regulatory and standards framework: overview and implications, in IEEE International Conference on UltraWideband, ICUWB 2007, Singapore, Singapore 733-738 (2007)

21. JA Paradiso, T Starner, Energy scavenging for mobile and wireless electronics. IEEE Pervas Comput. 4, 18-26 (2005)

22. Z Zou, M Baghaei-Nejad, H Tenhunen, L-R Zheng, An efficient passive RFID system for ubiquitous identification and sensing using impulse UWB radio, in e \& i Elektrotechnik und Informationstechnik, Springer Wien. 124(11), 397-403 (November 2007). doi:10.1007/s00502-007-0483-y

23. J Reunamaki, Ultra wideband radio frequency identification techniques. U.S. Patent 7,154,396, 26 Dec 2006

24. D Dardari, Method and apparatus for communication in ultra-wide bandwidth RFID systems. International Patent Application PCT/IB2009/000 360, (Feb. 25, 2009)

25. D Dardari, R D'Errico, Passive ultrawide bandwidth RFID. in IEEE Global Communications Conference (GLOBECOM 2008), New Orelans, LA, USA (November 2008)

26. F Guidi, D Dardari, C Roblin, A Sibille, in Backscatter communication using ultrawide bandwidth signals for RFID applications, ed. by Giusto D, et al The Internet of Things: 20th Tyrrhenian Workshop on Digital Communications, Pula, Sardinia, Italy (Springer-Science+BusinessMedia, 2009), pp. 251-262

27. SELECT (Smart and Efficient Location, identification, and Cooperation Techniques) in FP7 European SELECT Project http://www.selectwireless.eu

28. RC Hansen, Relationship between antennas as scatters and as radiators. Proc IEEE. 77(5), 659-662 (1989). doi:10.1109/5.32056

29. K Penttila, M Keskilammi, L Sydanheimo, M Kivikoski, Radar cross-section analysis for passive RFID systems, in IEE Proc Microwave and Antennas Propagation. 153(1), 103-109 (February 2006). doi:10.1049/ip-map:20045183

30. D Kim, M Ingram, W Smith, Small-scale fading for an indoor wireless channel with modulated backscatter, in Vehicular Technology Conference, 2001. VTC 2001 Fall. IEEE VTS 54th. 3, 1616-1620 (2001)

31. J Griffin, G Durgin, Link envelope correlation in the backscatter channel. Commun Lett IEEE. 11(9), 735-737 (2007)

32. S Hu, CL Law, Z Shen, L Zhu, W Zhang, W Dou, Backscattering cross section of ultrawideband antennas. IEEE Anten Wirel Propagat Lett. 6, 70-72 (2007)

33. S Hu, Y Zhou, CL Law, W Dou, Study of a uniplanar monopole antenna for passive chipless UWB-RFID localization system. IEEE Trans Anten Propagat. 58(2), 271-278 (2010)

34. F Guidi, A Sibille, D Dardari, C Roblin, UWB RFID backscattered energy in the presence of nearby metallic reflectors, in European Conference on Antennas and Propagation 2011, Rome, Italy (April 2011)

35. C Roblin, S Bories, A Sibille, Characterization tools of antennas in the time domain, in Proceedings of International Workshop on Ultra Wideband Band Systems (IWUWBS 2003) 
36. S Bories, H Ghannoum, C Roblin, Robust planar stripline monopole for UWB terminal applications, in 2005 IEEE International Conference on UltraWideband, pp. 80-84 (2005)

37. J Langley, P Hall, P Newham, Balanced antipodal Vivaldi antenna for wide bandwidth phased arrays, in IEE Proc on Microwave and Antennas Propagation. 143(2), (April 1996)

38. A Molisch, K Balakrishnan, D Cassioli, C-C Chong, S Emami, A Fort, J Karedal, J Kunisch, H Schantz, U Schuster, K Siwiak, IEEE 802.15.4a channel model. Final report, 2005.

39. C Xu, CL Law, TOA estimator for UWB backscattering RFID system with clutter suppression capability. EURASIP J Wirel Commun Netw. 2010, Article ID 753129, 1-14 (2010)

40. W Suwansantisuk, MZ Win, Multipath aided rapid acquisition: optimal search strategies. IEEE Trans Inf Theory. 52(1), 174-193 (2007)

41. D Dardari, E Falletti, M Luise, Satellite and Terrestrial RadioPositioning Techniques-A Signal Processing Perspective (Elsevier Ltd., London, 2011)

42. M Pursley, D Sarwate, W Stark, Error probability for direct-sequence spreadspectrum multiple-access communications. Part l: upper and lower bounds. IEEE Trans Commun. 30(5), 975-984 (1982). doi:10.1109/TCOM.1982.1095553

43. M Saito, $\mathrm{H}$ Yamamoto, Sequence assignment of Walsh-Hadamard sequences for quasi-synchronous multi-code CDMA systems, in The 5th International Symposium on Wireless Personal Multimedia Communications, 2002, vol. 2. (Honolulu, Hawaii, USA, October 2002), pp. 673-677

44. MZ Win, RA Scholtz, Characterization of ultra-wide bandwidth wireless indoor communications channel: a communication theoretic view. IEEE J Sel Areas Commun. 20(9), 1613-1627 (2002). doi:10.1109/JSAC.2002.805031

45. RK Mallik, MZ Win, Analysis of hybrid selection/maximal-ratio combining in correlated Nakagami fading. IEEE Trans Commun. 50(8), 1372-1383 (2002). do: $10.1109 / T C O M M .2002 .801495$

46. R D'Errico, A Sibille, A Giorgetti, M Chiani, Antenna diversity in UWB indoor channel, in IEEE International Conference on Ultra-Wideband, 2008 (ICUWB 2008). 2, 13-16 (September 2008)

doi:10.1186/1687-1499-2011-47

Cite this article as: Dardari et al.: Ultra-wide bandwidth backscatter modulation: processing schemes and performance. EURASIP Journal on Wireless Communications and Networking 2011 2011:47.

\section{Submit your manuscript to a SpringerOpen ${ }^{\mathcal{O}}$ journal and benefit from:}

- Convenient online submission

- Rigorous peer review

- Immediate publication on acceptance

- Open access: articles freely available online

- High visibility within the field

- Retaining the copyright to your article

Submit your next manuscript at $\gg$ springeropen.com 